\title{
Epigenetic markers to further understand insulin resistance
}

\author{
Charlotte Ling $^{1}$ Tina Rönn ${ }^{1}$
}

Received: 19 August 2016 / Accepted: 31 August 2016/Published online: 20 September 2016

(C) Springer-Verlag Berlin Heidelberg 2016

\begin{abstract}
Epigenetic variation in human adipose tissue has been linked to type 2 diabetes and its related risk factors including age and obesity. Insulin resistance, a key risk factor for type 2 diabetes, may also be associated with altered DNA methylation in visceral and subcutaneous adipose tissue. Furthermore, linking epigenetic variation in target tissues to similar changes in blood cells may identify new blood-based biomarkers. In this issue of Diabetologia, Arner et al studied the transcriptome and methylome in subcutaneous and visceral adipose tissue of 80 obese women who were either insulin-sensitive or -resistant (DOI 10.1007/s00125-016-4074-5). While they found differences in gene expression between the two groups, no alterations in DNA methylation were found after correction for multiple testing. Nevertheless, based on nominal $p$ values, their methylation data overlapped with methylation differences identified in adipose tissue of individuals with type 2 diabetes compared with healthy individuals. Differential methylation of these overlapping $\mathrm{CpG}$ sites may predispose to diabetes by occurring already in the insulin-resistant state. Furthermore, some methylation changes may contribute to an inflammatory process in adipose tissue since the identified $\mathrm{CpG}$ sites were annotated to genes encoding proteins involved in inflammation. Finally, the methylation pattern in circulating leucocytes did not mirror the adipose tissue methylome of these 80 women. Together, identifying novel molecular mechanisms contributing to insulin resistance and type 2 diabetes may help advance the search for new therapeutic alternatives.
\end{abstract}

Charlotte Ling

charlotte.ling@med.lu.se

1 Epigenetics and Diabetes Unit, Department of Clinical Sciences, Lund University Diabetes Centre, Scania University Hospital, Jan Waldenströms gata 35, 20502 Malmö, Sweden
Keywords Adipose tissue - Biomarker · DNA methylation · Epigenetics $\cdot$ EWAS $\cdot$ Insulin resistance $\cdot$ Subcutaneous · Type 2 diabetes $\cdot$ Visceral

\section{Abbreviations \\ DNMT DNA (cytosine-5)-methyltransferase \\ PBMC Peripheral blood mononuclear cell}

Insulin resistance is a condition where the cellular response to insulin is impaired, resulting in elevated insulin levels in the fasting state, although glucose levels are normal or elevated. It affects multiple tissues and is believed to be an underlying cause of type 2 diabetes. It is also a contributing factor for cardiovascular diseases, explained by the association with, for example, obesity, hypertension and dyslipidaemia [1]. Thereby, to prevent and treat many of our global metabolic diseases, we need to further understand insulin resistance on a molecular level.

As insulin resistance is caused by multiple factors and affects multiple tissues, there is a need to investigate the interactions between, for example, genetic and non-genetic factors and between different tissues. Epigenetic mechanisms, including DNA methylation, are strong candidates for mediating the environmental effect on the genome and are linked to gene activity and function [2]. In differentiated human cells, DNA methylation mainly takes place on a cytosine residue followed by guanine, a so called $\mathrm{CpG}$ site. Key enzymes regulating DNA methylation include DNA (cytosine-5)-methyltransferase (DNMT) $3 \mathrm{~A}$ and $-3 \mathrm{~B}$, which are involved in de novo methylation, as well as DNMT1, which copies the methylation patterns during replication. In addition, the ten-eleven translocation (TET) enzymes were found to be involved in active demethylation by oxidising methyl groups into 
hydroxymethyl. An inactive demethylation process may also take place during replication, if the enzyme activity of DNMT or the levels of methyl donors are low. Increased DNA methylation was initially thought to reduce gene transcription. However, it is now well established that DNA methylation has numerous different functions depending on the location in the genome and can subsequently be associated with both decreased and increased gene expression, as well as alternative splicing events and genome stability [3]. It has important functions during cell differentiation, and regulates cell-specific gene expression, imprinting and $\mathrm{X}$ chromosome inactivation in females.

Unlike genetic variation, the epigenome is largely tissue- or even cell-specific, requiring the correct target tissue and/or cell samples for analyses [4]. The involvement of epigenetic factors in insulin resistance is also supported by the theory of a metabolic memory, linking an impaired intrauterine environment to increased risk for insulin resistance later in life [5].

A diminished effect of insulin sensing in adipose tissue is known to increase hydrolysis of triacylglycerols, which further reinforces insulin resistance and thereby increase the level of circulating insulin. In addition, ectopic lipid accumulation in peripheral tissues and low grade inflammation in adipose tissue are important characteristics of insulin resistance [1]. In the current study by Arner et al, the epigenetic signature of systemic insulin resistance was studied in subcutaneous and visceral adipose tissue, to investigate whether there is a link between insulin resistance and DNA methylation [6]. The authors also characterised the gene expression profile in adipose tissue of the same cohort, including 80 obese women who were selected in either of the extreme ends of the distribution curve of insulin sensitivity based on HOMA-IR. It should be noted that, although both groups included obese women, the insulin-resistant group had a significantly higher $\mathrm{BMI}$ and greater waist circumference compared with the more insulin-sensitive group. There has been an increasing interest in linking epigenetic signatures in human tissues to those in blood cells, to potentially identify blood-based epigenetic biomarkers that predict disease [7]. In the present study, the authors examined whether the epigenetic pattern in peripheral blood mononuclear cells (PBMCs) mirrors DNA methylation in adipose tissue and could potentially be used as a marker for insulin resistance.

As previous studies have linked adipose tissue DNA methylation to both BMI and type 2 diabetes [8-10], it is reasonable to assume an association also with insulin resistance. However, after correction for multiple testing, Arner and colleagues were unable to find significant associations between the investigated methylation sites and insulin resistance in this cohort. Nevertheless, they present thousands of differentially methylated sites based on a nominal association, and validate their results by showing that some of them are also found in similar studies of DNA methylation in adipose tissue related to BMI, type 2 diabetes or weight loss [6, 9-11]. For example, 591 differentially methylated sites identified in adipose tissue of individuals with type 2 diabetes compared with nondiabetic controls in a study by Nilsson et al were also found to be nominally associated with insulin resistance in the study by Arner et al $[6,9]$. These include sites annotated to $I L 18$, CD44, CD48, CD 38, CD37, CX3CL1, CXCR1, CXCR2, CXCL1, IGF1R, APOB48R, LEF1, GIPR, GRB10, SIRT2, HDAC4, DNMT3A, LEPR and LEP (encoding leptin). It is possible that altered methylation of these sites predisposes to diabetes by occurring already in the insulin-resistant state, and methylation changes in some of these genes may contribute to an inflammatory process in the adipose tissue. In addition, Arner et al report an overlap of 1973 sites in which DNA methylation in adipose tissue of insulin-resistant women is directionally consistent with the BMI-associated methylation of $\mathrm{CpG}$ sites identified in a study by Rönn et al $[6,10]$. Since the insulin-resistant women in the Arner et al study also have a higher BMI, it is difficult to conclude whether these 1973 sites are primarily associated with higher BMI rather than insulin sensitivity. It should also be noted that Arner et al found 91 differentially methylated sites that overlapped with sites that changed methylation in adipose tissue after weight loss induced by bariatric surgery $[6,11]$. Whether differential methylation of these sites contributes to improved insulin sensitivity needs to be further tested.

In addition, the study by Arner and colleagues identifies 223 genes in subcutaneous and 18 genes in visceral adipose tissue that have both differential gene expression and methylation sites with a nominal difference, in insulin-resistant compared with insulin-sensitive women. Only a few of them could be verified by a direct methylation-expression correlation. Interestingly, the findings in subcutaneous fat highlight several genes involved in insulin signalling. Arner et al also found altered expression of genes related to the immune response and angiogenesis [6]. Looking at the transcriptomic data presented in this study as a whole, there were considerably more insulin resistance-related alterations in subcutaneous than visceral adipose tissue.

Arner and colleagues also try to answer the question of whether there is a preference for negative correlations between differential DNA methylation and gene expression in the $5^{\prime}$ region of the genes, and a positive correlation within the gene body, which has previously been hypothesised [3]. This seems not to be the case in the current study; however, this sub-analysis was limited to include only genes differentially expressed between adipose tissue from insulin-resistant and -sensitive women rather than the whole dataset. Furthermore, to arrive at a more definite conclusion it would have been interesting to take into account gene expression levels, as functions of DNA methylation may differ between highly or lowly expressed genes, or maybe affected by the presence of a $\mathrm{CpG}$ island in the promoter region.

Finally, DNA methylation was investigated in PBMCs to see whether this could be used as a surrogate marker for the 
adipose tissue methylome. However, in this cohort of obese women, circulating leucocytes did not mirror the adipose tissue methylome [6]. Considering that the nominally associated methylation sites in PBMCs were fewer than expected by chance, this result needs to be validated in a larger cohort. The inability to confirm an association here should not be confused with an established lack of association.

Sample size is always an issue when using genome-wide arrays, and especially for detection of the small differences in DNA methylation, where tissue specificity and the availability of tissues of interest are clearly limiting factors. This study only interrogates $0.3-0.4 \%$ of $\mathrm{CpG}$ methylation sites in the whole genome and, moreover, the comparison between tissues becomes even more limited as the filtering and exclusion of probes was done individually for each tissue.

As in many association studies, there is a drawback in not knowing whether the epigenetic alterations observed mediate insulin resistance or are secondary to the condition, and it is difficult to investigate this further by carrying out longitudinal studies on human tissues other than blood. Regardless of which is the case, there may be a use for the identified epigenetic markers in diagnostics, but if it is found that the alterations are secondary to insulin resistance, their use in prevention or treatment is less pronounced. Importantly, the degree of methylation in adipose tissue also seems to mediate the impact of common genetic variants (single nucleotide polymorphisms [SNPs]) on metabolic traits, including insulin sensitivity measured by HOMA-IR [12]. Hence, further studies examining how interactions between genetics and epigenetics affect insulin sensitivity may identify additional important loci for disease development.

For future studies, more $\mathrm{CpG}$ sites need to be investigated, as regional methylation patterns may be more indicative of transcriptional activity than single sites. As the epigenome is much more complex than the simple addition of methyl groups to DNA, the many different histone modifications also need to be analysed and integrated to further understand the epigenetic impact on insulin resistance and other phenotypes. It would also be interesting to know if the results would be similar in insulin-resistant but normal weight individuals, or transferable to males, considering different body composition between sexes.

In summary, this genome-wide study by Arner et al identified transcriptional alterations of genes involved in the immune response, insulin signalling and angiogenesis in adipose tissue of insulin-resistant compared with insulin-sensitive obese women. The overlap between their epigenetic findings and those of other studies suggest a role for DNA methylation in the development of insulin resistance and type 2 diabetes. Overall, an improved understanding of the contribution of epigenetic mechanisms to the development of insulin resistance, type 2 diabetes and related complications may help to improve future prediction, prevention and treatment of these conditions.

Funding This work was funded by grants from the Swedish Research Council, Region Skåne and The Swedish Diabetes Foundation.

Duality of interest The authors declare that there is no duality of interest associated with this manuscript.

Contribution statement All authors were responsible for drafting the article and revising it critically for important intellectual content. All authors approved the version to be published.

\section{References}

1. Samuel VT, Shulman GI (2016) The pathogenesis of insulin resistance: integrating signaling pathways and substrate flux. J Clin Invest 126:12-22

2. Ling C, Groop L (2009) Epigenetics: a molecular link between environmental factors and type 2 diabetes. Diabetes 58:2718-2725

3. Jones PA (2012) Functions of DNA methylation: islands, start sites, gene bodies and beyond. Nat Rev Genet 13:484-492

4. Ziller MJ, Gu H, Muller F et al (2013) Charting a dynamic DNA methylation landscape of the human genome. Nature 500:477-481

5. Gillberg L, Perfilyev A, Brons C et al (2016) Adipose tissue transcriptomics and epigenomics in low birthweight men and controls: role of high-fat overfeeding. Diabetologia 59:799-812

6. Arner P, Sahlqvist AS, Sinha I et al (2016) The epigenetic signature of systemic insulin resistance in obese women. Diabetologia. doi:10.1007/s00125-016-4074-5

7. Bacos K, Gillberg L, Volkov P et al (2016) Blood-based biomarkers of age-associated epigenetic changes in human islets associate with insulin secretion and diabetes. Nat Commun 7:11089

8. Dick KJ, Nelson CP, Tsaprouni L et al (2014) DNA methylation and body-mass index: a genome-wide analysis. Lancet 383:19901998

9. Nilsson E, Jansson PA, Perfilyev A et al (2014) Altered DNA methylation and differential expression of genes influencing metabolism and inflammation in adipose tissue from subjects with type 2 diabetes. Diabetes 63:2962-2976

10. Rönn T, Volkov P, Gillberg L et al (2015) Impact of age, BMI and $\mathrm{HbA}_{1 \mathrm{c}}$ levels on the genome-wide DNA methylation and mRNA expression patterns in human adipose tissue and identification of epigenetic biomarkers in blood. Hum Mol Genet 24:3792-3813

11. Benton MC, Johnstone A, Eccles D et al (2015) An analysis of DNA methylation in human adipose tissue reveals differential modification of obesity genes before and after gastric bypass and weight loss. Genome Biol 16:8

12. Volkov P, Olsson AH, Gillberg L et al (2016) A genome-wide mQTL analysis in human adipose tissue identifies genetic variants associated with DNA methylation, gene expression and metabolic traits. PLoS One 11:e157776 